\title{
Overview of hard hadron production results in ALICE
}

\author{
Dmitri Peresunko ${ }^{1, *}$ for the ALICE collaboration \\ ${ }^{1}$ NRC Kurchatov institute, Kurchatov sq.,1, 123182, Moscow
}

\begin{abstract}
The ALICE experiment is designed to study the properties the hot and dense medium, the Quark-Gluon Plasma (QGP), produced in ultrarelativistic heavy-ion collisions at the LHC. Measuring production of hadrons with large $Q^{2}$ transfer in these collisions provides the possibility to explore one of the most spectacular effects - the in-medium parton energy loss. By varying the observables among light and heavy flavored hadrons and fully reconstructed jets and by changing the colliding systems from $\mathrm{pp}$ to $\mathrm{p}-\mathrm{Pb}$ and $\mathrm{Pb}-\mathrm{Pb}$, one can explore the transport properties of hot matter in great details. Here an overview of recent ALICE results on high- $p_{\mathrm{T}}$ hadron and jet production in $\mathrm{pp}, \mathrm{p}-\mathrm{A}$ and A-A collisions at LHC energies is presented.
\end{abstract}

\section{Introduction}

The Large Hadron Collider (LHC) provides opportunity to study hadron production in different colliding systems: pp, p-A and A-A and at several colliding energies. This allows us to check our understanding of hadron production in $\mathrm{pp}$ collisions and to study modifications related to the creation of quark-gluon plasma (QGP) in A-A collisions. In addition it allows investigating the origin of the collective-like effects spotted in high multiplicity pp and $\mathrm{p}-\mathrm{Pb}$ collisions. Approximately, the hard hadron production in $\mathrm{pp}$ collisions can be considered as a superposition of a process with high- $Q^{2}$ transfer and some underlying event. The hard hadron spectrum in this approach is the convolution of proton structure functions (PDF), cross-section of elementary process and fragmentation function (FF). However, as it became clear recently [1], at least in high multiplicity pp collisions this picture is too simplified and has to be extended to include collective effects like long-range correlations [2], features similar to collective flow [3] and enhancement of strangeness production [4].

Similarly, the hard hadron production in p-A collisions can be approximated as a set of independent nucleon-nucleon collisions supplemented with interactions of final particles with cold nuclear matter. Comparing these data with pp collisions, one can study modifications of nucleon structure functions in nuclei and cold nuclear matter effects. Similar to pp collisions, collective effects were found in high multiplicity p-A collisions [5], and what is more surprising, a smooth continuation of $\mathrm{pp}$ data as a function of charged particles multiplicity was observed $[4,6]$.

Heavy-ion collisions can approximately be described as a hydrodynamic-like expansion of hot matter with hard partons interacting with it. A-A collisions provide possibility to study properties of hot matter and details of interaction of hard partons with it: average energy loss, path-length dependence and a modification of fragmentation process.

\footnotetext{
*e-mail: Dmitri.Peresunko@cern.ch
} 
Below the recent ALICE results on identified hard hadron and jet production in $\mathrm{pp}, \mathrm{p}-\mathrm{A}$ and A-A collisions are presented and discussed.

\section{ALICE setup}

ALICE experiment is one of four large experiments at the Large Hadron Collider. As its primary physics objective is the study of properties of quark-gluon matter created in heavyion collisions, it was designed to be able to work in high multiplicity environment [7]. Thanks to the relatively low magnetic field $B=0.5 \mathrm{~T}$ and tiny material budget of the inner detectors, ALICE central tracking system is able to track particles with transverse momentum down to $p_{\mathrm{T}} \sim 100 \mathrm{MeV} / c$.

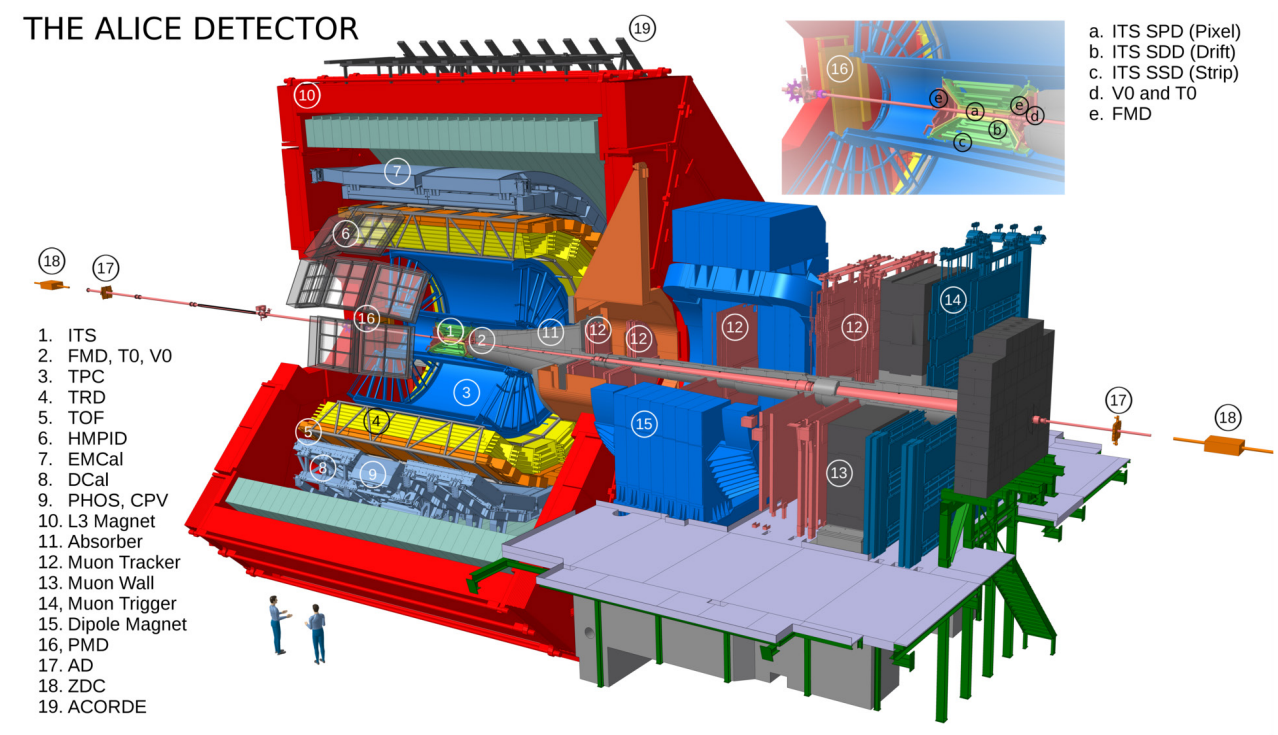

Figure 1. Setup of the ALICE experiment in 2015-2018 data taking period.

ALICE apparatus includes a central barrel, a muon arm and a set of detectors used for triggering and event characterization, see Fig. 1. Central barrel includes Inner Tracking System [8], Time Projection Chamber [9], Transition Radiation detector [10], Time Of Flight [11] and Cerenkov detector HMPID [12]. In addition ALICE has two electromagnetic calorimeters, EMCAL+DCAL [13] with large acceptance but modest energy and position resolutions and PHOS [14] with small acceptance but excellent resolutions.

ALICE setup includes detectors exploiting all available particle identification techniques [15]: the specific energy loss of charged particles is measured in ITS and TPC detectors. They provide particle identification at low $p_{\mathrm{T}} \lesssim 0.7 \mathrm{GeV} / c$ and also at $3 \lesssim p_{\mathrm{T}} \lesssim 20 \mathrm{GeV} / c$ based on statistical separation in the region of relativistic rise. The TOF detector provides $3 \sigma$ $\pi / K$ separation up to $p_{\mathrm{T}} \sim 2.5 \mathrm{GeV} / c$ and $K / p$ separation up to $p_{\mathrm{T}} \sim 4 \mathrm{GeV} / c$. The Cerenkov detector HMPID extends $p_{\mathrm{T}}$ range of particle separation on the $3 \sigma$ level to $p_{\mathrm{T}} \sim 3 \mathrm{GeV} / c$ for the $\pi / K$ and $p_{\mathrm{T}} \sim 5 \mathrm{GeV} / c$ for the $K / p$ separation. In addition electrons can be identified in TRD. Finally, the topological particle identification is applied in wide $p_{\mathrm{T}}$ range. 


\section{3 pp collisions}

ALICE measured spectra of large variety of hadron species at all colliding energies provided by LHC. We present spectra of neutral pions and $\eta$-mesons [16-19] in Fig. 2. Each of these results combines 4 to 7 independent measurements, where photons are measured either in calorimeter PHOS or EMCAL or reconstructed as electron-positron pair after conversion on the material of inner detectors or their combination. This allows us to extend the $p_{\mathrm{T}}$ range and significantly reduce systematic uncertainties. Comparison to Pythia and NLO pQCD predictions illustrates the typical situation for light unflavored hadrons: Pythia approximately reproduces spectra while NLO calculations predict higher yield: $20-50 \%$ for neutral pions and about factor 2 for $\eta$-mesons. These observations suggest that current parameterization of PDF and FF can be improved by inclusion of the latest LHC results into global fits.
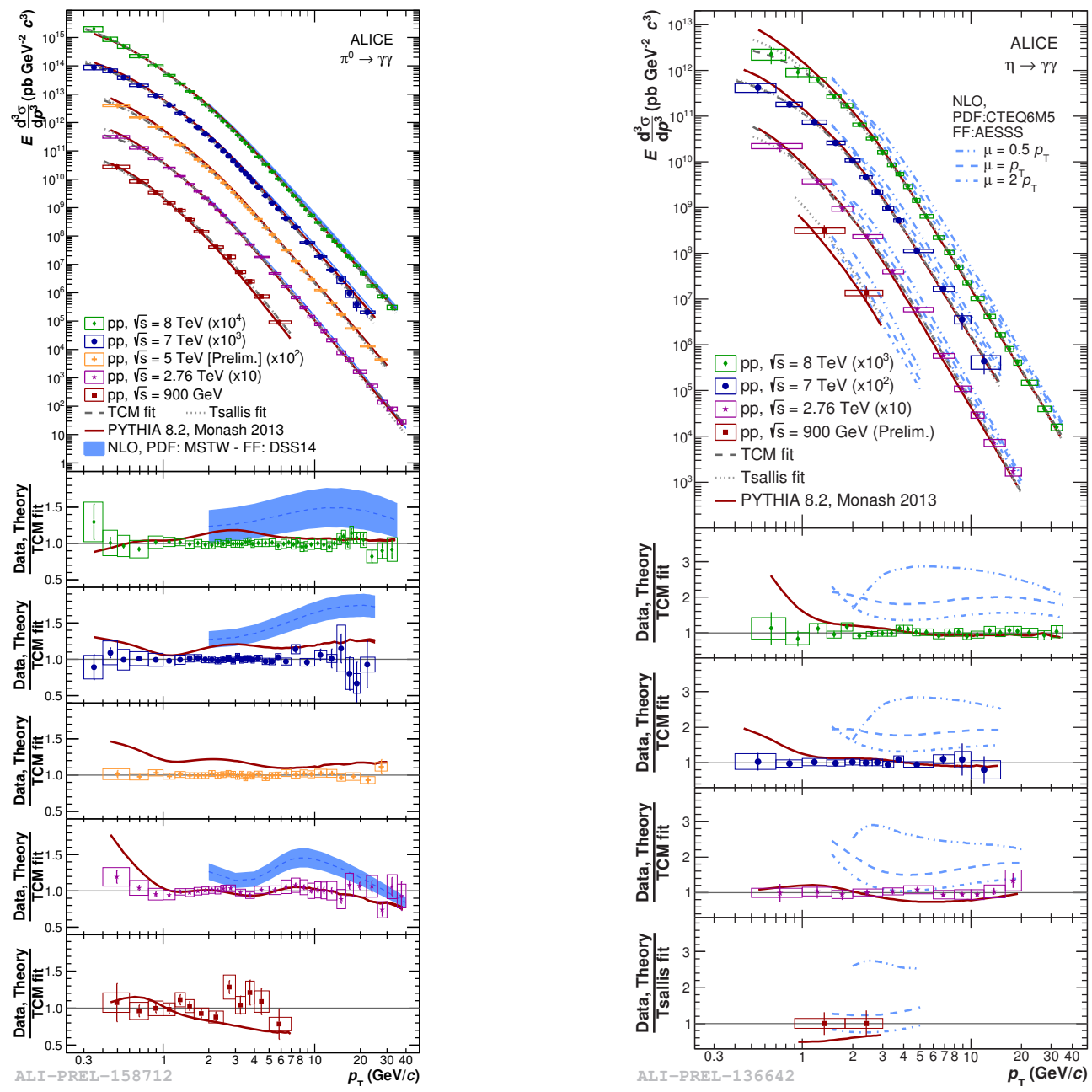

Figure 2. Neutral pion (left) and $\eta$-meson spectra measured in pp collision at several collision energies compared to predictions of Pythia and NLO pQCD calculations. Bottom part of the plots show the ratio of measured spectra and predictions to the fit with Two Component Model (TCM) function. 

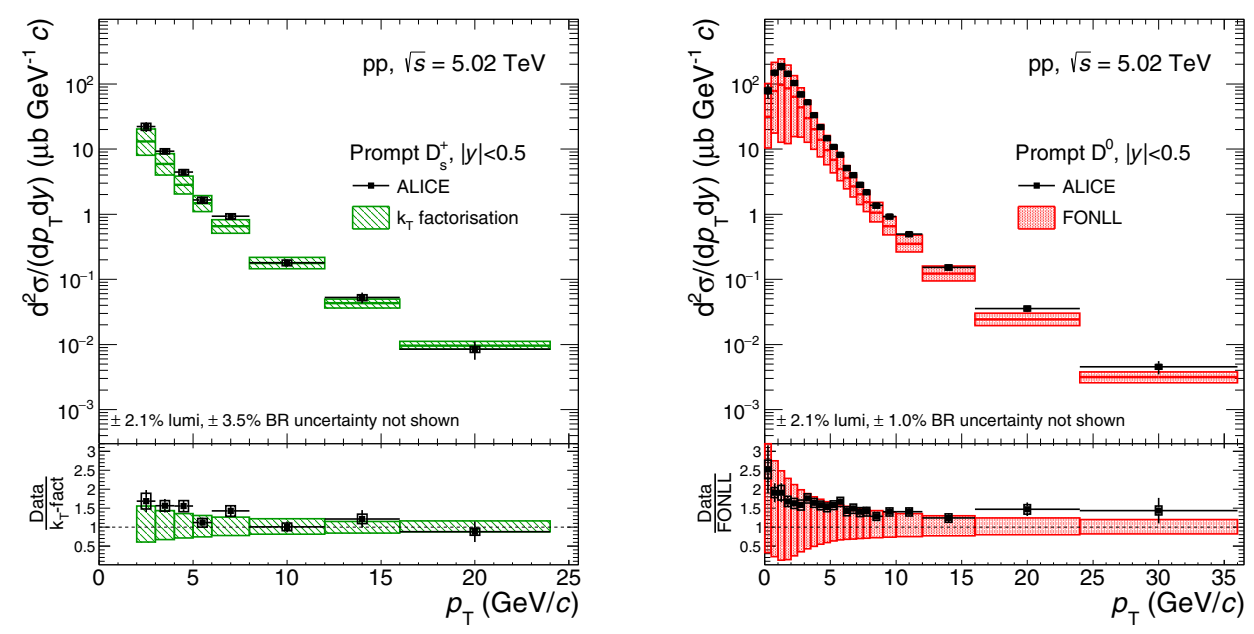

Figure 3. Invariant cross section of prompt $\mathrm{D}_{\mathrm{s}}^{+}($left $)$and $\mathrm{D}^{0}$ (right) production in pp collisions at $\sqrt{s}=$ $5.02 \mathrm{TeV}$ in comparison with $\mathrm{k}_{\mathrm{T}}$-factorization (left) and FONLL (right) calculations [20].

In addition to light flavored hadrons, ALICE has measured spectra of hadrons with heavy flavors. Production of hadrons with open charm is especially interesting because $c$-quarks are mostly produced in the processes with gluons in the initial state and thus probe the gluon PDF in the low- $x$ region, where presently it is poorly constrained. D-mesons were measured in different channels: $D^{0}, D^{+}, D^{*+}$ and $D_{s}^{+}$[20], see Fig. 3. The invariant production crosssection was compared to the predictions of several calculations, among them $k_{\mathrm{T}}$-factorization approach and FONLL. In contrast to light-flavored hadrons, both calculations predict smaller yield, although agree with data within uncertainties.

Large integrated luminosity of pp collisions collected at $\sqrt{s}=5$ and $13 \mathrm{TeV}$ allows for more differential measurements. Identified hadron spectra were measured in different classes of charged particles multiplicities [21]. Identified hadron spectra were measured at $|\eta|<0.8$ while while the charged particle multiplicity class was evaluated either using ITS detector $(|\eta|<0.8)$ or the $\mathrm{V} 0$ detector $\left(-3.7<\eta_{\mathrm{V} 0 \mathrm{C}}<-1.7\right.$ and $\left.2.8<\eta_{\mathrm{V} 0 \mathrm{~A}}<5.1\right)$. For the multiplicity dependence based on V0 multiplicity, a hardening of spectra in the region $0.5 \lesssim p_{\mathrm{T}}<3 \mathrm{GeV} / c$ in events with higher multiplicity is observed while at higher $p_{\mathrm{T}}$ shapes are similar. This multiplicity dependence is well reproduced by Pythia 6 and Pythia 8, where it can be attributed to the larger number of high- $p_{\mathrm{T}}$ jets in high multiplicity events.

One can get further inside into details of particle production in pp collisions by classifying events according to their shape using sphericity or similar variable which better handles 3-jet events - transverse spherocity, $S_{0}$, originally proposed in [22]. $S_{0}$ is defined for a unit vector $\hat{\mathbf{n}}_{\mathbf{s}}$ which minimizes the ratio:

$$
S_{0} \equiv \frac{\pi^{2}}{4} \min _{\hat{\mathbf{n}}_{\mathrm{s}}}\left(\frac{\sum_{i}\left|\vec{p}_{\mathrm{T}, i} \times \hat{\mathbf{n}}_{\mathrm{s}}\right|}{\sum_{i} p_{\mathrm{T}, i}}\right)^{2},
$$

where the sum runs over all reconstructed tracks. At least three tracks are required in order to achieve a good spherocity resolution. Small values of spherocity correspond to elongated or jetty event, while spherocity around unity means cylindrically symmetric events. To have good resolution of $S_{0}$, only $10 \%$ of highest multiplicity (VOMI-III classes) are considered in 

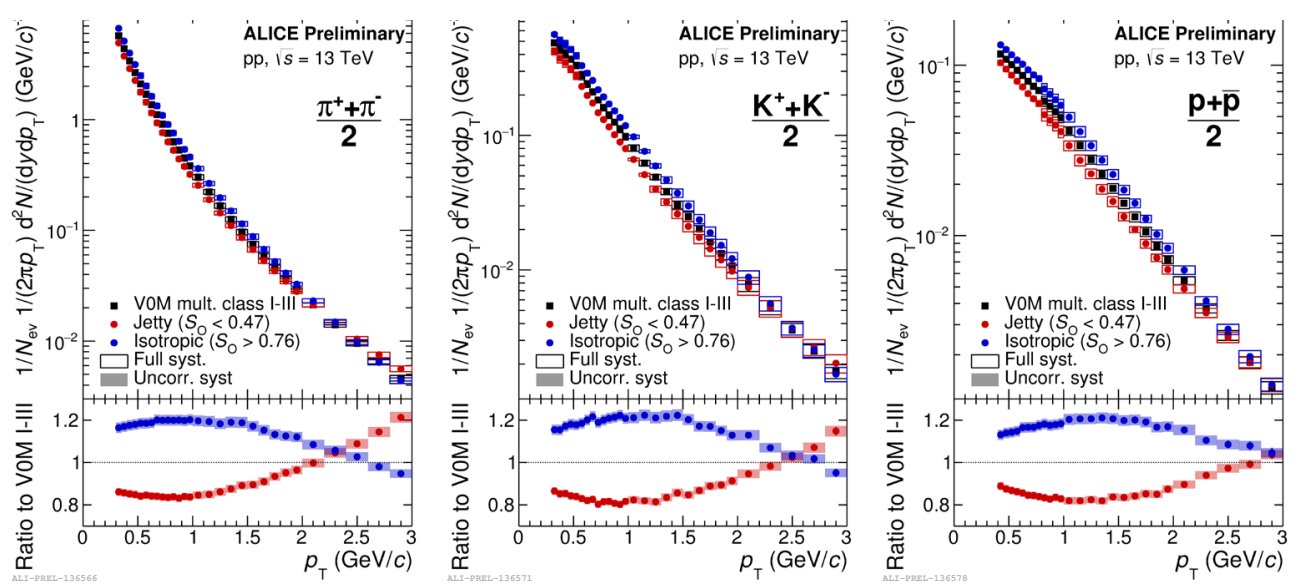

Figure 4. Top panels: transverse momentum spectra of pions (left), kaons (middle) and protons (right) in jetty events ( $20 \%$ lowest $S_{0}$ ) and isotropic events ( $20 \%$ highest $S_{0}$ ). Bottom panels: ratio of spectra in jetty and isotropic events to the $S_{0}$-integrated spectrum.

this analysis, see Fig. 4. Spectra of identified hadrons in symmetric events are softer than in jet-like and $p_{\mathrm{T}}$ of crossing point increases with the particle mass. Similar dependence one would observe in case of development of collective flow, therefore it can be considered as an indication of considerable contribution of multi-parton interactions in high multiplicity pp collisions.

\section{$4 \mathrm{p}-\mathrm{Pb}$ collisions}

In $\mathrm{p}$ - $\mathrm{Pb}$ collisions one can look at possible modifications of initial state, e.g. modification of nucleon structure functions in nuclei, and at interactions of final partons or hadrons with cold nuclear matter. For quantitative comparison it is convenient to construct a ratio $Q_{\mathrm{pPb}}$

$$
Q_{\mathrm{pPb}}=\frac{\mathrm{d} N^{\mathrm{pPb}} / \mathrm{d} y \mathrm{~d}^{2} p_{\mathrm{T}}}{\left\langle T_{\mathrm{pPb}}\right\rangle \mathrm{d} \sigma^{\mathrm{pp}} / \mathrm{d} y \mathrm{~d}^{2} p_{\mathrm{T}}},
$$

where $\mathrm{d} N^{\mathrm{pPb}} / \mathrm{d} y \mathrm{~d}^{2} p_{\mathrm{T}}$ is the hadron spectrum measured in $\mathrm{p}-\mathrm{Pb}$ collisions, $d \sigma^{\mathrm{pp}} / \mathrm{d} y \mathrm{~d}^{2} p_{\mathrm{T}}$ is the invariant hadron production cross-section in pp collisions at the same energy and $\left\langle T_{\mathrm{pPb}}\right\rangle$ is the nuclear thickness, calculated within geometrical Glauber model for given multiplicity class of p-Pb collisions. For unidentified charged particles [23], light-flavored [24] and heavy flavored [25] mesons the ratio at high $p_{\mathrm{T}} \gtrsim 6 \mathrm{GeV} / c$ is consistent with unity, meaning absence of significant modifications in the initial state and of strong interaction of final particles with cold nuclear matter.

As some signs of collective behavior was observed in high multiplicity pp collisions, it is interesting to explore multiplicity dependence of $\mathrm{p}-\mathrm{Pb}$ collisions as well. Nuclear modification factors $Q_{\mathrm{pPb}}$ were measured in several multiplicity classes. The observed modifications strongly depend on the rapidity gap between measured particles and those used to evaluate the multiplicity class [26]: the smaller rapidity gap, the stronger is multiplicity dependence. In Fig. $5 Q_{\mathrm{pPb}}$ for unidentified charged hadrons, light unflavored mesons and open charm mesons measured in $20 \%$ highest multiplicity and $40 \%$ lowest multiplicity collisions estimated using neutron Zero Degree Calorimeter (ZNA) detector located at $|\eta|>8.7$ in the 

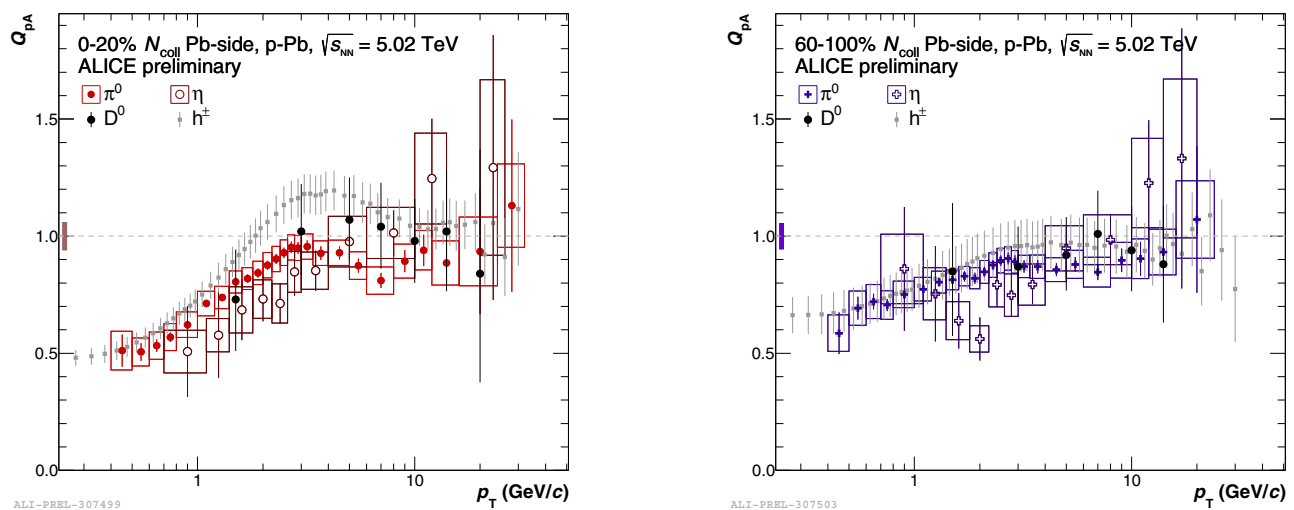

Figure 5. Nuclear modification factor of unidentified charged hadrons, light- and heavy flavored mesons, measured in $20 \%$ high multiplicity (left) and $40 \%$ lowest multiplicity (right) classes of p- $\mathrm{Pb}$ collisions at $\sqrt{s_{\mathrm{NN}}}=5.02 \mathrm{TeV}$.

direction of $\mathrm{Pb}$ beam as a multiplicity estimator is presented. At high $p_{\mathrm{T}} \gtrsim 6 \mathrm{GeV} / c$ the ratio is consistent with unity for all measured species meaning absence of modification of initial state in any of these channels. A bump at intermediate $p_{\mathrm{T}}$ observed for unidentified charged hadrons is related to the increase of baryon to meson ratio with increase of charged particles multiplicity [27] and is explained by combination of hydrodynamic [28] and recombination [29] models .

\section{$5 \mathrm{~Pb}-\mathrm{Pb}$ collisions}

To quantify modification of hadron spectra in A-A collisions a nuclear modification factor $R_{\mathrm{AA}}$ can be defined:

$$
R_{\mathrm{PbPb}}=\frac{1}{\left\langle T_{\mathrm{PbPb}}\right\rangle} \frac{\mathrm{d} N^{\mathrm{PbPb}} / \mathrm{d} y \mathrm{~d}^{2} p_{\mathrm{T}}}{\mathrm{d} \sigma^{\mathrm{pp}} / \mathrm{d} y \mathrm{~d}^{2} p_{\mathrm{T}}}=\frac{1}{\left\langle N_{\text {coll }}\right\rangle} \frac{\mathrm{d} N^{\mathrm{PbPb}} / \mathrm{d} y \mathrm{~d}^{2} p_{\mathrm{T}}}{\mathrm{d} N^{\mathrm{Pp}} / \mathrm{d} y \mathrm{~d}^{2} p_{\mathrm{T}}},
$$

where $\left\langle T_{\mathrm{PbPb}}\right\rangle$ is the nuclear thickness function and $\left\langle N_{\text {coll }}\right\rangle$ is the number of binary nucleonnucleon collisions in a given centrality class calculated with Glauber model. Three large LHC experiments, ALICE, ATLAS and CMS, provided large amount of data on unidentified and identified hadron production and reported suppression of hadron production in the most central $\mathrm{Pb}-\mathrm{Pb}$ collisions up to a factor $\sim 10$. An excellent agreement between $R_{\mathrm{AA}}$ measured by different experiments was found [30]. On the other hand, for colorless objects, $W^{ \pm}, Z^{0}[31]$ and isolated [32] and direct [33] photons no suppression within uncertainties was observed. ALICE measured direct photon spectra in three centrality classes of $\mathrm{Pb}-\mathrm{Pb}$ collisions and confirmed agreement with NLO pQCD calculations scaled with $N_{\text {coll }}$ down to $p_{\mathrm{T}} \sim 4 \mathrm{GeV} / c$, while at lower $p_{\mathrm{T}}$ a thermal direct photon radiation becomes significant. This all confirms that modification of the initial state in A-A collisions is minor if any, and suppression is the consequence of parton interaction with hot quark-gluon matter.

ALICE measured nuclear modification factors of light-flavored and strange hadrons [34, 35], vector mesons [36] and even $\rho$-mesons [37]. Within uncertainties all these species follow a common trend. An example of nuclear modification factor measured for neutral pions in $\mathrm{Pb}-\mathrm{Pb}$ collisions at $\sqrt{s_{\mathrm{NN}}}=5.02 \mathrm{TeV}$ is shown in Fig. 6. Suppression up to a factor $\sim 10 \mathrm{in}$ the most central collisions is found. Magnitude of suppression decreases with centrality and 


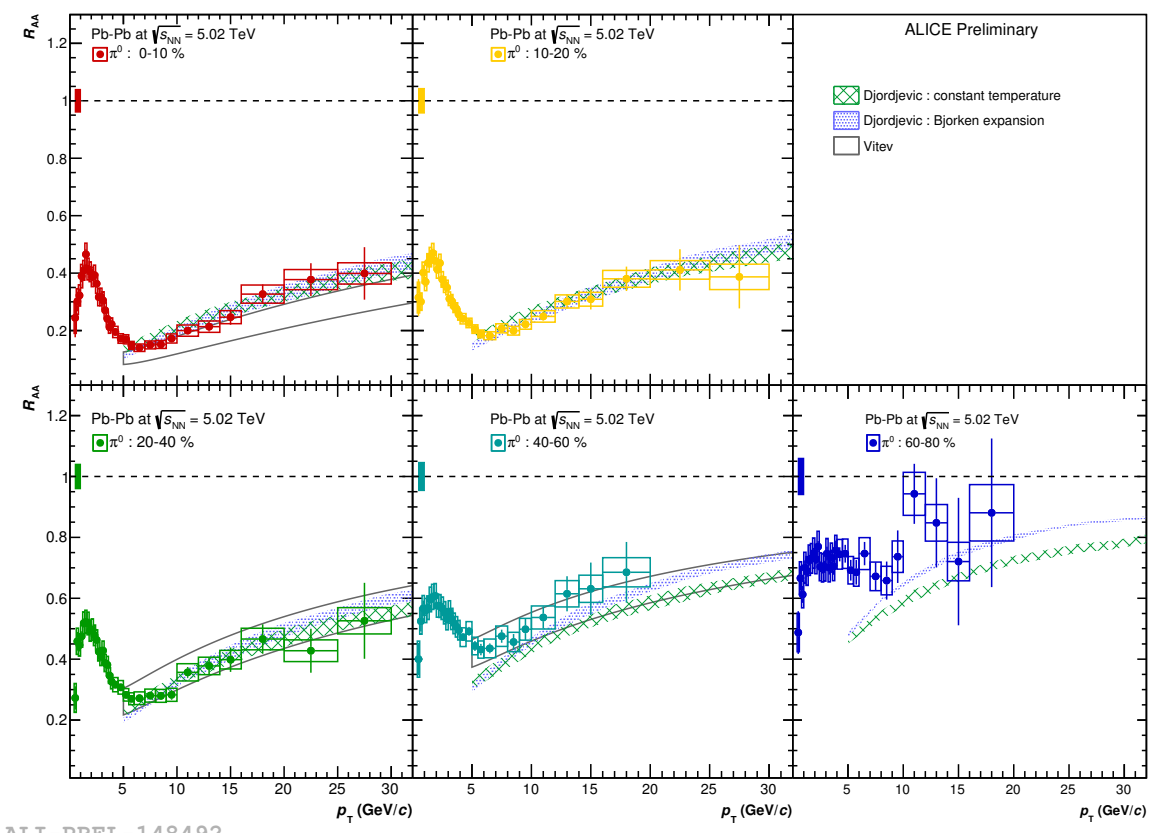

ALI-PREL-148492

Figure 6. Nuclear modification factor $R_{\mathrm{AA}}$ of neutral pions measured in several centrality classes of $\mathrm{Pb}-\mathrm{Pb}$ collisions at $\sqrt{s_{\mathrm{NN}}}=5.02 \mathrm{TeV}$, compared to predictions of two models accounting energy loss of a parton in hot matter.

reaches $0.7-0.8$ in the most peripheral centrality class. Theoretical calculations of Djorjevich [38] and Vitev [39] take into account light parton energy loss in hot quark-gluon matter and reproduce both absolute value of the suppression and its $p_{\mathrm{T}}$ and centrality dependence.
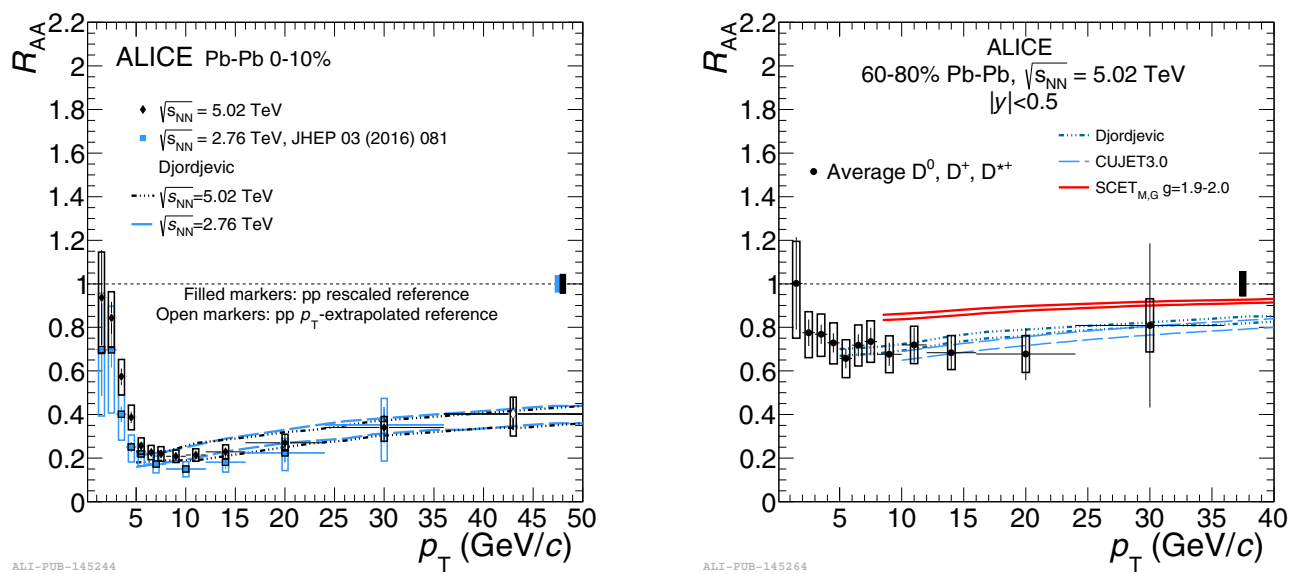

Figure 7. Nuclear modification factor $R_{\mathrm{AA}}$ of D-mesons measured in $0-10 \%$ most central and 60$80 \%$ most peripheral centrality classes of $\mathrm{Pb}-\mathrm{Pb}$ collisions at $\sqrt{s_{\mathrm{NN}}}=2.76$ and $5.02 \mathrm{TeV}$, compared to predictions of models accounting energy loss of parton in hot matter. 
Naively one can expect that energy loss of heavy quarks is smaller because of deadcone effect and therefore, suppression of e.g. D-mesons should be smaller. Surprisingly, the measured $R_{\mathrm{AA}}$ of D-mesons (see Fig. 7) appeared to be similar to that of light flavored hadrons at high $p_{\mathrm{T}} \gtrsim 8 \mathrm{GeV} / c$, while at intermediate $p_{\mathrm{T}}$ suppression of D-mesons is smaller. There are several possible effects, starting from difference in energy loss of light and heavy quarks, to recombination or initial state effects. Comparing measured $R_{\mathrm{AA}}$ for neutral pions and Dmesons to the calculations of the Djorjevich [40] model, one founds that it is able to reproduce both cases. This model accounts for different energy loss of light and heavy quarks, but this difference is compensated by the harder $p_{\mathrm{T}}$ distribution and the harder fragmentation function of charm quarks compared to those of light quarks and gluons. This situation resembles similarity of $R_{\mathrm{AA}}$ measured at RHIC and LHC energies: despite the very different energy loss of partons, the observed suppressions are similar. When one goes to the heavier b-quark, the described cancellation does not happen any more and a clear ordering of suppression with quark mass is observed [41].

More detailed study of the parton energy loss in hot matter can be preformed using azimuthal asymmetry of high- $p_{\mathrm{T}}$ hadron production with respect to reaction plane azimuthal angle $\Psi_{\mathrm{RP}}$. At low $p_{\mathrm{T}}$ this asymmetry originates due to hydrodynamic-like expansion of initially asymmetric fireball created by overlap of colliding nuclei. In contrast, at high $p_{\mathrm{T}}$ the asymmetry appears because of difference in path length in different directions of the fireball, asymmetric at early stages. The asymmetry is decomposed into Fourier series

$$
\mathrm{d} N / \mathrm{d}\left(\varphi-\Psi_{\mathrm{RP}}\right)=N\left(1+2 v_{1} \cos \left(\varphi-\Psi_{\mathrm{RP}}\right)+2 v_{2} \cos \left(2\left(\varphi-\Psi_{\mathrm{RP}}\right)\right)+\ldots\right)
$$

The elliptic flow coefficient $v_{2}$ was measured for $\mathrm{D}$-mesons in mid-central $\mathrm{Pb}-\mathrm{Pb}$ collisions, see Fig. 8, where it is expected to be maximal. Fig. 8 shows simultaneous comparison of measured $R_{\mathrm{AA}}$ and $v_{2}$ coefficient with predictions of different models. Most of available models are able to simultaneously reproduce both suppression and asymmetry at high $p_{\mathrm{T}}$, though some of them fails: e.g. BAMPS model with elastic energy loss approximately reproduces $v_{2}$ but fails to reproduce $R_{\mathrm{AA}}$, BAMPS with elastic and radiative energy loss reproduces better $R_{\mathrm{AA}}$ but fails in $v_{2}$. LIDO and DAB-MOD M\&T models predict somewhat smaller $v_{2}$.
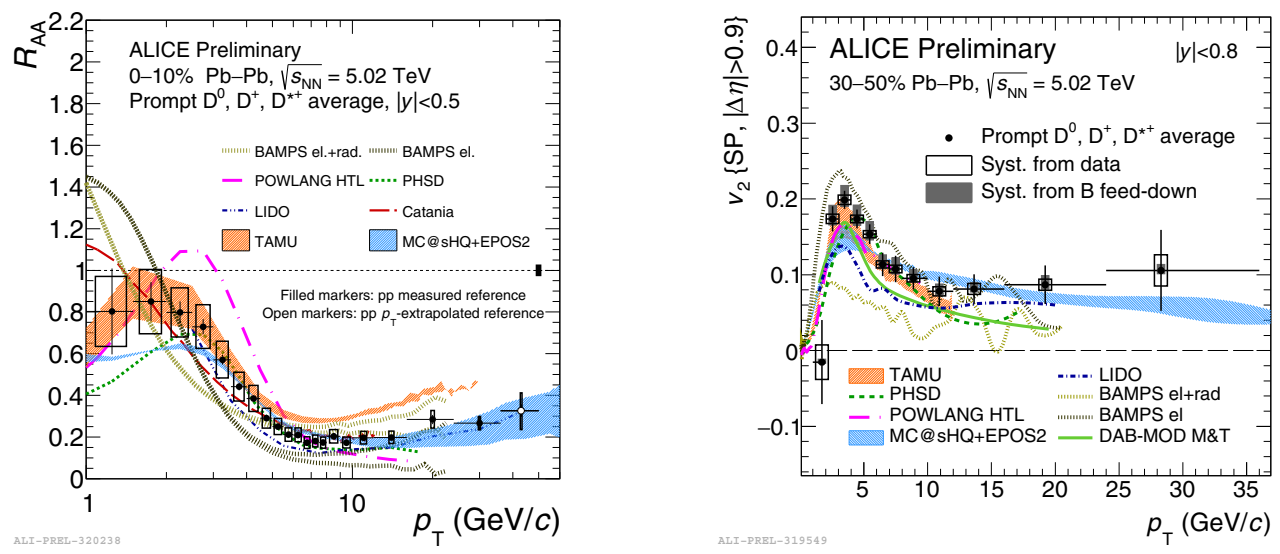

Figure 8. Left plot: Nuclear modification factor of $\mathrm{D}$-mesons, measured in $\mathrm{Pb}-\mathrm{Pb}$ collisions at $\sqrt{s_{\mathrm{NN}}}=$ $5.02 \mathrm{TeV}$, compared to predictions of several event generators. Right plot: azimuthal asymmetry in D-meson production in mid-central $\mathrm{Pb}-\mathrm{Pb}$ collisions at $\sqrt{s_{\mathrm{NN}}}=5.02 \mathrm{TeV}$, compared to predictions of several event generators. 


\section{Jet modifications}

Measurement of fully reconstructed jets provides possibility to explore parton energy loss from another point of view and to check if there is a modification in parton fragmentation process in hot matter. To produce a baseline, ALICE measured jet spectra in pp collisions at $\sqrt{s}=2.76,5,7 \mathrm{TeV}[42-45]$ and $13 \mathrm{TeV}$, see Fig. 9. For jet reconstruction the anti- $k_{\mathrm{T}}$ algorithm was used with radius parameter $R=0.4$. The POWHEG+PYTHIA8 calculations agree with measured spectra within uncertainties - similar to single light flavored hadron spectra.

Spectra of jets tagged with charm quark (D-meson) were also measured in pp collisions at several energies, $\sqrt{s}=7$ [46] and $13 \mathrm{TeV}$, see Fig. 9. POWHEG with heavy quark implementation + PYTHIA8 calculations reproduce measured spectrum within uncertainties, similar to spectra of single hadrons with c quark.
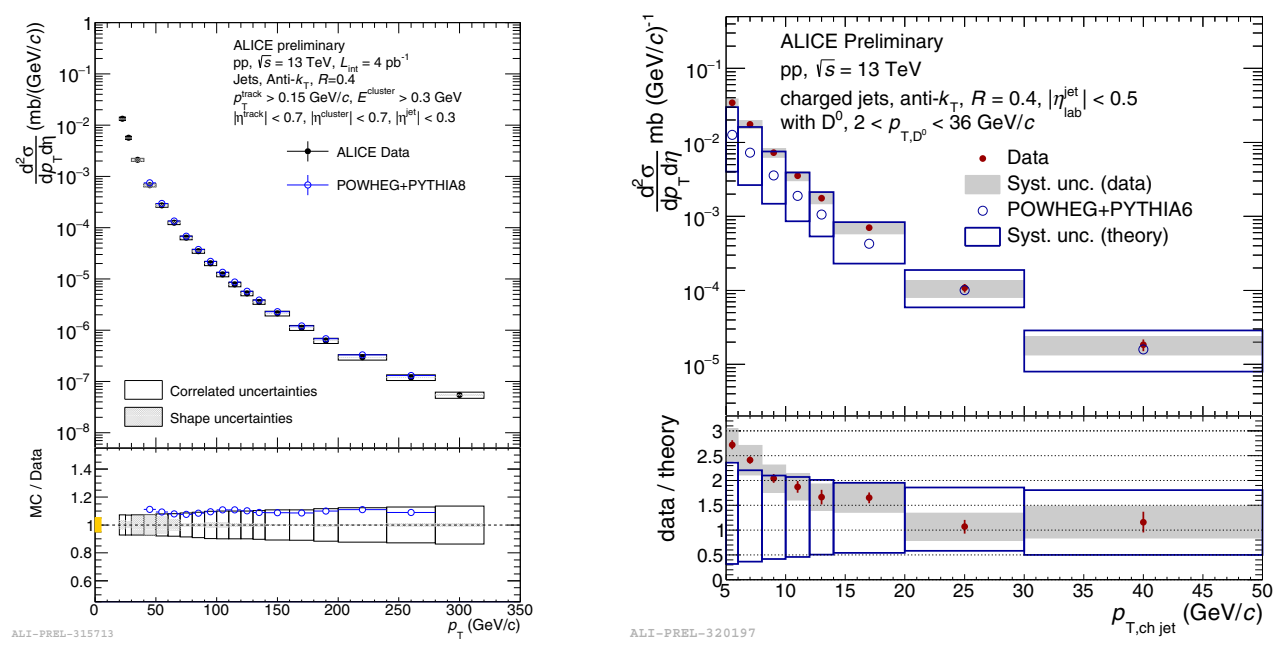

Figure 9. Left: Differential production cross section of jets in pp collisions at $\sqrt{s}=13 \mathrm{TeV}$ compared to POWHEG+PYTHIA8. Right: Differential production cross section of jets tagged with a $D^{0}$ meson with $p_{T, D}>2 \mathrm{GeV} / c$ in pp collisions at $\sqrt{s}=13 \mathrm{TeV}$.

Exploring modification of jets in A-A collisions, one should consider two qualitatively different situations: if gluon radiation happens inside the jet cone, suppression of jet spectrum will be limited, while emission beyond the cone radius would cause strong suppression of jet yield. First measurements of jets with radius parameter $R=0.2$ [47] demonstrated that jet suppression is comparable with one of single hadrons, at least for this jet radius. It is interesting to find, at which jet radii parameter the gluon emission will be recovered. The main problem of jet analysis in A-A collisions is a big background with large fluctuations, distorting reconstructed jet energy resolution and resulting in appearance of fake jets. Increase of the jet radius parameter $R$ rapidly increases amount of fluctuations. Recently ALICE developed technique based on Machine Learning (ML) to estimate the contribution of the background event. This technique uses simulated PYTHIA jets embedded to the real event as a signal for training. This approach allows to reduce fluctuations of underlying event more than $\sim 2$ times and to apply large jet radii to explore the gluon radiation. Using this technique the nuclear modification factor of jet with radius parameter from $R=0.2$ to $R=0.6$ was measured in central $\mathrm{Pb}-\mathrm{Pb}$ collisions at $\sqrt{s_{\mathrm{NN}}}=5 \mathrm{TeV}$, see Fig. 10 . For the small jet radius $R=0.2$ one 

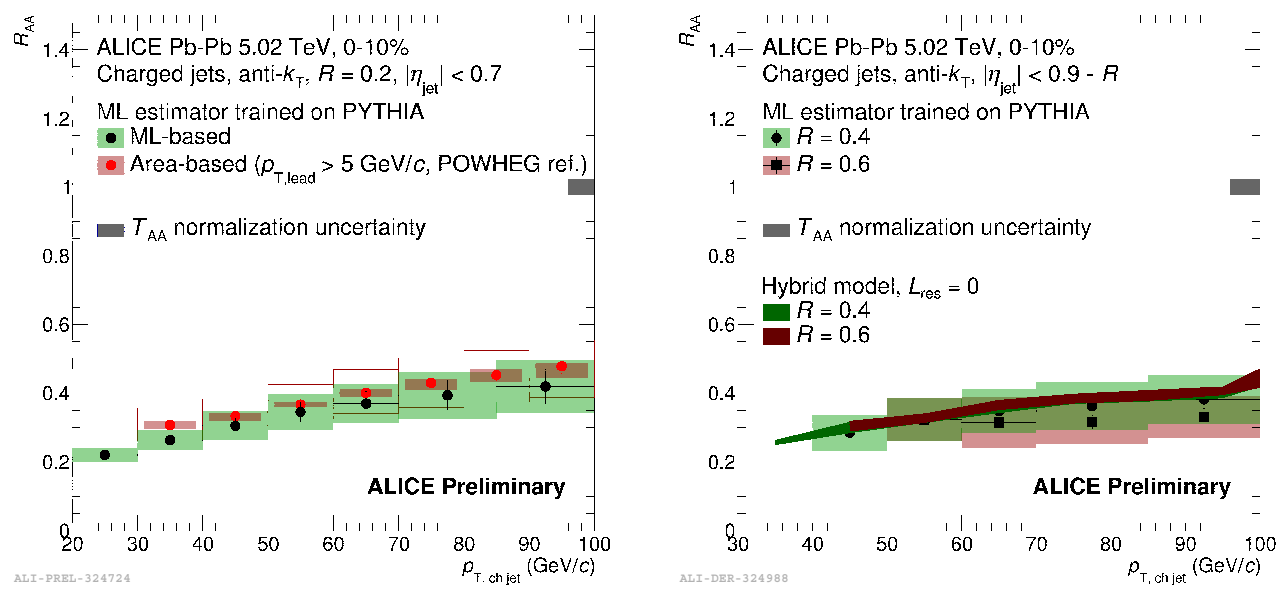

Figure 10. Charged jet nuclear modification factor $R_{\mathrm{AA}}$ measured in $10 \%$ of the most central $\mathrm{Pb}-\mathrm{Pb}$ collisions at $\sqrt{s_{\mathrm{NN}}}=5 \mathrm{TeV}$. Left plot: comparison of $R_{\mathrm{AA}}$ calculated with area-based and ML-based corrections. Right plot: comparison of $R_{\mathrm{AA}}$ measured for jets with $R=0.4$ and $R=0.6$ using ML-based correction.

can compare the measured $R_{\mathrm{AA}}$ with the one produced with standard area-based background subtraction, see Fig. 10, left plot. Good agreement between two methods is found. Comparing suppression of jets with two radius parameters, $R=0.4$ and $R=0.6$, see Fig. 10, right plot, similar suppression in both cases is found. This can mean either emission at large angles or emission of very soft gluons, not recovered in jet reconstruction.

Modification of jets in A-A collisions can be explored in different ways, looking at modification of jet mass [48], or exploring their modification in longitudinal or transverse directions. Jet dispersion in transverse direction can be described with girth variable, defined as

$$
g \equiv \frac{\sum_{\text {tracks }} p_{\mathrm{T}, \mathrm{i}} r}{p_{\mathrm{T}, \mathrm{jet}}} .
$$
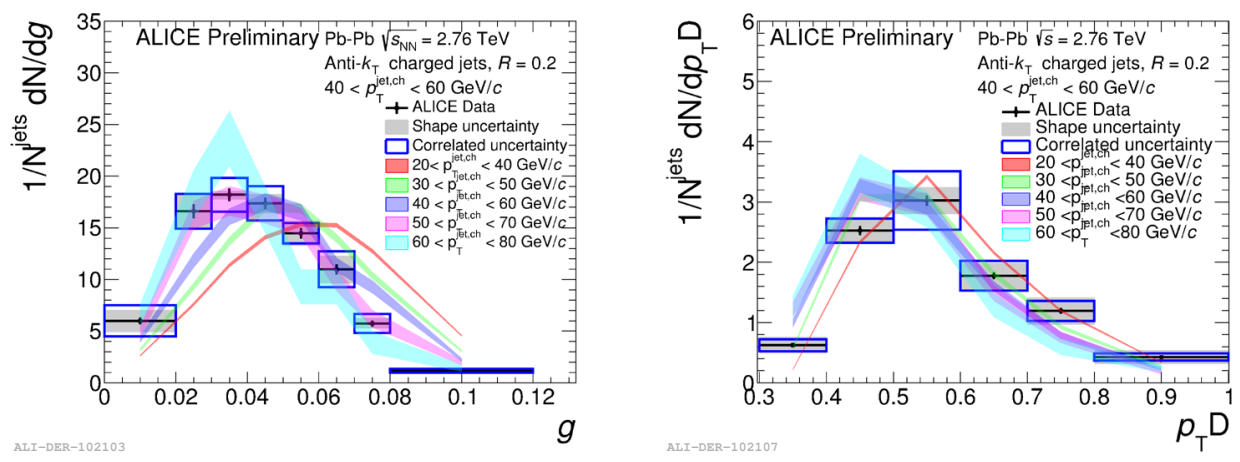

Figure 11. Distributions over girth $g$ (left) and $p_{\mathrm{T}} D$ of charged jets with $R=0.2$ measured in $\mathrm{Pb}-\mathrm{Pb}$ collisions at $\sqrt{s_{\mathrm{NN}}}=2.76 \mathrm{TeV}$ compared to Pythia with different jet $p_{\mathrm{T}}$. 
Dispersion of jet in longitudinal direction can be characterized with $p_{\mathrm{T}} D$ variable, defined as:

$$
p_{\mathrm{T}} D \equiv \frac{\sum_{\text {tracks }} p_{\mathrm{T}, \mathrm{i}}^{2}}{p_{\mathrm{T}, \mathrm{jet}}}
$$

Distribution of jets over these two variables measured in $\mathrm{Pb}-\mathrm{Pb}$ collisions at $\sqrt{s_{\mathrm{NN}}}=$ 2.76 TeV is shown in Fig. 11 and compared to the PYTHIA6 simulations (Perugia tune) with different jet $p_{\mathrm{T}}$ in order to study the $p_{\mathrm{T}}$ evolution of the jet shape. Radial moment $g$ in $\mathrm{Pb}-\mathrm{Pb}$ collisions is found to be smaller than in pp (PYTHIA) and longitudinal dispersion $p_{\mathrm{T}} D$ in $\mathrm{Pb}-\mathrm{Pb}$ collisions is larger than in pp (PYTHIA).

\section{Conclusions}

A review of recent ALICE results on hard hadron and jet production in pp, $\mathrm{p}-\mathrm{A}$ and $\mathrm{A}-\mathrm{A}$ collisions was presented. Spectra of unidentified charged particles and of identified hadrons were measured in pp collisions at several energies provided by LHC. Comparison of measured spectra with NLO pQCD calculations shows that for light-flavored hadrons calculation usually predicts significantly higher yields, while PYTHIA event generator approximately reproduce the spectra. Spectra of hadrons with heavy quarks are better reproduced with pQCD calculations. Probably, parameterizations of structure and fragmentation functions can be improved by inclusion of the LHC results to the global QCD fits.

Hard hadron spectra measured in $\mathrm{p}$ - $\mathrm{Pb}$ collisions are not modified with respect to spectra measured in pp collisions at the same energy and scaled with the number of binary nucleonnucleon collisions - except the spectra of baryons which reflect increase of baryon/meson ratio at intermediate $p_{\mathrm{T}}$ with increase of charged particle multiplicity.

Hadron production in $\mathrm{Pb}-\mathrm{Pb}$ collisions reflect strong suppression, related to the parton energy loss in hot quark-gluon matter. At high $p_{\mathrm{T}}$, suppression of light-flavored hadrons and hadrons with c-quark appears to be similar. This similarity is probably a result of accidental approximate cancellation of higher energy loss of light quarks and harder spectrum. Hadrons with b-quarks restore ordering and show smaller suppression.

Measurements of fully reconstructed jets in pp collisions show good agreement of measured spectra and calculations with POWHEG+PYTHIA model. A strong suppression of jets in $\mathrm{Pb}-\mathrm{Pb}$ collisions was observed, similar to the suppression of yield of single hadrons. Similarity of suppression of jets, reconstructed with small radius parameter $R=0.2$ and large $R=0.6$ suggests that gluon emission happens at very large angle. Finally some modification of jet shape in $\mathrm{Pb}-\mathrm{Pb}$ collisions is observed: transverse dispersion becomes smaller, while longitudinal dispersion increases.

This research was supported by the Russian Science Foundation grant 17-72-20234.

\section{References}

[1] K. Dusling, W. Li, B. Schenke, Int. J. Mod. Phys. E25, 1630002 (2016), 1509. 07939

[2] V. Khachatryan et al. (CMS), JHEP 09, 091 (2010), 1009. 4122

[3] B.B. Abelev et al. (ALICE), Phys. Lett. B726, 164 (2013), 1307. 3237

[4] J. Adam et al. (ALICE), Nature Phys. 13, 535 (2017), 1606.07424

[5] B. Abelev et al. (ALICE), Phys. Lett. B719, 29 (2013), 1212. 2001

[6] S. Acharya et al. (ALICE), Phys. Rev. Lett. 123, 142301 (2019), 1903.01790

[7] International Journal of Modern Physics A 29, 1430044 (2014)

[8] K. Aamodt et al. (ALICE), JINST 3, S08002 (2008) 
[9] J. Alme et al., Nucl. Instrum. Meth. A622, 316 (2010), 1001.1950

[10] S. Acharya et al. (ALICE), Nucl. Instrum. Meth. A881, 88 (2018), 1709.02743

[11] F. Carnesecchi (ALICE), JINST 14, C06023 (2019), 1806.03825

[12] G. De Cataldo (ALICE), Nucl. Instrum. Meth. A876, 62 (2017)

[13] U. Abeysekara et al. (ALICE EMCal) (2010), 1008.0413

[14] G. Dellacasa et al. (ALICE) (1999)

[15] B.B. Abelev et al. (ALICE), Int. J. Mod. Phys. A29, 1430044 (2014), 1402 . 4476

[16] B. Abelev et al. (ALICE), Phys. Lett. B717, 162 (2012), 1205 . 5724

[17] B.B. Abelev et al. (ALICE), Eur. Phys. J. C74, 3108 (2014), 1405 . 3794

[18] S. Acharya et al. (ALICE), Eur. Phys. J. C77, 339 (2017), [Eur. Phys. J.C77,no.9,586(2017)], 1702 .00917

[19] S. Acharya et al. (ALICE), Eur. Phys. J. C78, 263 (2018), 1708.08745

[20] S. Acharya et al. (ALICE), Eur. Phys. J. C79, 388 (2019), 1901.07979

[21] S. Acharya et al. (ALICE) (2019), 1905.07208

[22] A. Banfi, G.P. Salam, G. Zanderighi, JHEP 06, 038 (2010), 1001.4082

[23] B.B. Abelev et al. (ALICE), Eur. Phys. J. C74, 3054 (2014), 1405. 2737

[24] J. Adam et al. (ALICE), Phys. Lett. B760, 720 (2016), 1601.03658

[25] S. Acharya et al. (ALICE) (2019), 1906.03425

[26] J. Adam et al. (ALICE), Phys. Rev. C91, 064905 (2015), 1412.6828

[27] S. Acharya et al. (ALICE), Phys. Rev. C99, 024906 (2019), 1807. 11321

[28] H. Song, U.W. Heinz, Phys. Rev. C78, 024902 (2008), 0805 . 1756

[29] V. Minissale, F. Scardina, V. Greco, Phys. Rev. C92, 054904 (2015), 1502.06213

[30] B. Abelev et al. (ALICE), Phys. Lett. B720, 52 (2013), 1208. 2711

[31] S. Chatrchyan et al. (CMS), Phys. Lett. B715, 66 (2012), 1205.6334

[32] S. Chatrchyan et al. (CMS), Phys. Lett. B710, 256 (2012), 1201. 3093

[33] J. Adam et al. (ALICE), Phys. Lett. B754, 235 (2016), 1509.07324

[34] J. Adam et al. (ALICE), Phys. Rev. C93, 034913 (2016), 1506.07287

[35] S. Acharya et al. (ALICE) (2019), 1910.07678

[36] J. Adam et al. (ALICE), Phys. Rev. C95, 064606 (2017), 1702.00555

[37] S. Acharya et al. (ALICE), Phys. Rev. C99, 064901 (2019), 1805.04365

[38] M. Djordjevic, M. Djordjevic, Phys. Lett. B734, 286 (2014), 1307. 4098

[39] I. Vitev, Phys. Lett. B639, 38 (2006), hep-ph/0603010

[40] M. Djordjevic, M. Djordjevic, B. Blagojevic, Phys. Lett. B737, 298 (2014), 1405 . 4250

[41] J. Adam et al. (ALICE), Phys. Lett. B771, 467 (2017), 1609.07104

[42] B. Abelev et al. (ALICE), Phys. Lett. B722, 262 (2013), 1301. 3475

[43] B.B. Abelev et al. (ALICE), Phys. Rev. D91, 112012 (2015), 1411.4969

[44] S. Acharya et al. (ALICE), Phys. Rev. D99, 012016 (2019), 1809. 03232

[45] S. Acharya et al. (ALICE) (2019), 1905.02536

[46] S. Acharya et al. (ALICE), JHEP 08, 133 (2019), 1905.02510

[47] J. Adam et al. (ALICE), Phys. Lett. B746, 1 (2015), 1502 . 01689

[48] S. Acharya et al. (ALICE), Phys. Lett. B776, 249 (2018), 1702.00804 